\title{
Substituents interplay in piperidinyl-perylenediimide as dopant-free hole-selective layer for perovskite solar cells fabrication
}

\author{
David Payno ${ }^{1} \cdot$ Manuel Salado ${ }^{1}$. Michael Andresini ${ }^{1} \cdot$ David Gutiérrez-Moreno ${ }^{2}$. Peng Huang ${ }^{1} \cdot$ Fulvio Ciriaco ${ }^{3}$. \\ Samrana Kazim ${ }^{1,4} \cdot$ Ángela Sastre-Santos $^{2}$ · Fernando Fernández-Lázaro² ${ }^{2} \cdot$ Shahzada Ahmad $^{1,4}$
}

Received: 12 May 2021 / Accepted: 14 October 2021 / Published online: 30 November 2021

(c) The Author(s) 2021

\begin{abstract}
The charge selective layer is of significance for the fabrication of emerging photovoltaics, including perovskite-based solar cells. Molecular hole transport materials (HTMs) are being employed as charge transporters, owing to their synthetic molecular flexibility that allows the fine-tuning of their electro-optical properties. Typically, doping of HTMs is essential, but it is a trade-off between long-term durability and device performance. The energetic level of perylenediimides (PDIs) was altered by the position of the substituent. The substituent's position influences the geometry of the PDI core, which can lose planarity, thus presenting a core twist angle between the two naphthalene subunits to find its application as hole-selective layers for fabrication. We have fabricated perovskite solar cells, with pristine PDI, and it gave a competitive performance. New design protocols for PDIs are required for aligned energetic levels, which will minimize recombination losses in solar cells, favoring a performance enhancement.
\end{abstract}

Keywords Photovoltaics $\cdot$ Perovskite solar cells $\cdot$ Hole-selective layer $\cdot$ Perylenediimides

\section{Introduction}

Perylenediimides (PDIs) have received significant attention as dyes, charge selective layers in electro-optical devices, two-photon-absorbing chromophores, fluorophores, and acceptors in photoinduced electron-transfer reactions. The use of PDIs was also investigated as light-harvesting

David Payno, Manuel Salado, Michael Andresini and David Gutiérrez-Moreno contributed equally to this work.

Fernando Fernández-Lázaro

fdofdez@umh.es

Shahzada Ahmad

shahzada.ahmad@bcmaterials.net

1 BCMaterials-Basque Center for Materials, Applications and Nanostructures, Martina Casiano, UPV/EHU Science Park, Barrio Sarriena S/N, 48940 Leioa, Spain

2 Área de Química Orgánica, Instituto de Bioingeniería, Universidad Miguel Hernández, Avda. Universidad S/N, 03202 Elche, Spain

3 Department of Chemistry, University of Bari “A. Moro" Via E, Orabona 4, 70125 Bari, Italy

4 IKERBASQUE, Basque Foundation for Science, 48013 Bilbao, Spain electron-transporting materials in organic solar cells owing to their comparable electron affinities to fullerenes, excellent photochemical stability, and tuneable electronic properties [1].

PDIs possess high thermal, chemical, and optical stabilities [2]. Moreover, some of their physicochemical properties such as solubility, electron affinity, and absorbance and emission wavelengths can be easily tuned by introducing substituents in their structure [3, 4]. Thus, functionalization at $1,6,7$, and 12 locations of the aromatic core, aptly called "bay" positions, was noted to have a deep influence on the optical and redox properties. Depending on the substituents on the bay positions, PDIs can behave as electron-accepting or as electron-donating molecules. The possibility of finetuning through the aromatic core functionalization has made PDIs attractive for their application in optoelectronic devices [5-9], and in organic, or perovskite solar cells (PSCs) [10].

Recently, a PDI has been reported as electron-transporting material (ETM) for PSC fabrication, as a cost-effective alternative to the commonly used fullerene derivative [6,6]-phenyl- $\mathrm{C}_{61}$-butyric acid methyl ester (PCBM) [11-14]. Fluorinated PDIs at the imide positions used as ETM also improved the air, moisture, and thermal stability of PSCs achieving efficiencies as high as $23.80 \%$ together with

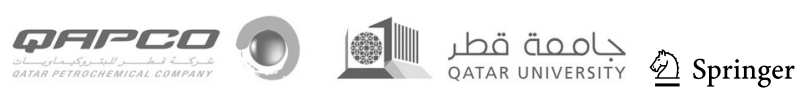


long-term stability [15-17]. Moreover, a disubstituted PDI with thiophene groups at the bay positions was employed as hole transport material (HTM) in PSCs affording a PCE of 5.6\% [18]. Taking into consideration the requirements for cost-effective materials, the ease of fine-tuning by functionalization in the bay positions to yield low-lying highest occupied molecular orbital (HOMO) levels will put forward the PDIs as alternative HTMs.

In the present report, we have investigated the 1,7 and 1,6 isomers of dipiperidin- $N$-yl-substituted PDI, named PDI-1 and PDI-2, respectively. They can be straightforwardly synthesized through a metal-free approach [19-27]. The modification by the substituents twists the PDI core, which strongly affects its optoelectronic properties [3, 28]. The introduction of a strong electron-donating group at bay positions confers higher frontier molecular orbital (FMO) energy levels to the reported thiophene-substituted PDI. We unravel the influence of the substitution at bay positions of the PDIs on the preparation of materials. Furthermore, by computational DFT-B3LYP calculations with a 6-311G* basis set, we complemented its energetic level as well as orientation studies.

\section{Results and discussion}

To shed light on PDI distribution of frontier orbitals, which facilitates charge exchange processes, we performed computational DFT-B3LYP calculations with a 6-311G* basis set. The resulting HOMO and LUMO for the optimized structures mainly interest the perylene core and both piperidinyl substituents at bay positions, while frontier orbitals are not distributed on substituents linked to the imide groups (Fig. 1). The presence of substituents in 1,7 (PDI-1) and 1,6 (PDI-2) positions strongly influences the geometry of the perylene core that loses planarity and presents a core twist angle between the two naphthalene subunits. Figure 2 represents the dihedral angles between $\mathrm{C}_{6}$ and $\mathrm{C}_{7}$. As a result, piperidinyl substitution on 1,6 and 1,7 positions causes a similar deformation of the perylene core: the optimized geometries for PDIs show a twist angle of $18.6^{\circ}$ for PDI-1 and $20.4^{\circ}$ for PDI-2, which are in agreement with a previous report of DFT-B3LYP 6-311G** calculations [29]. While electronic properties of substituents on 1,6 and 1,7 positions are reported to strongly affect HOMO-LUMO levels, steric hindrance of the fragment linked to the bay positions could bias frontier orbitals energies by affecting the core twist angle and subsequently aromatic resonance stabilization energy along with the perylene core.

Spiro-OMeTAD is the most studied HTM; its hole mobility and extended purification steps contribute to its cost, while it demands doping to increase carrier concentration for its application in PSCs. The ease of synthesis for PDIs (Fig. 3a), and opportunity for functionalization through molecular chemistry, suggests their suitability as HTMs. The values of HOMO and LUMO levels calculated by DFT are useful as the first approach, but not all the parameters that influence the energetic levels of a film are taken into account. Cyclic voltammetry (CV) measurements were carried out (SI, Figure S1), and the obtained HOMO values for PDI-1 $(-5.06 \mathrm{eV})$ and PDI-2 $(-5.22 \mathrm{eV})$ were noted above that of the methylammonium lead halide perovskite $\left[\mathrm{MAPbI}_{3}(\mathrm{HOMO} \approx-5.43 \mathrm{eV}\right.$ and LUMO $\approx-3.93 \mathrm{eV})$, indicating their suitability for hole extraction.

The scanning electron microscopy image (Fig. 3d) of PDI-2 deposited on bare glass shows a compact microstructure. The inset of Fig. 3d depicts the visual image of PDI deposited on FTO glass.

The conductivity and charge carrier mobility was calculated by fabricating asymmetric devices with a structure (ITO/HTL/Ag), and the linear current density-voltage $(J$ $V$ ) curve under dark and ambient conditions (Fig. 4a) was measured. The conductivity $\left(\sigma_{0}\right)$ value of pristine PDI-2 was found to be $\sim 0.72 \mu \mathrm{S} \mathrm{cm} \mathrm{cm}^{-1}$ derived from the equation $\sigma_{0}=J \cdot L \cdot V^{-1}$ ( $L$ : the thickness of HTL, $J$ : current density, $V$ : applied voltage). Furthermore, we used the spacecharge limited current (SCLC) method to evaluate the mobility using a hole-only device with a structure of ITO/ PEDOT:PSS/HTL/Ag (Fig. 4b). The mobility $(\mu)$ of pristine PDI-2 was found to be $\sim 7.56 \times 10^{-8} \mathrm{~cm}^{2} \mathrm{~V}^{-1} \cdot \mathrm{s}^{-1}$ calculated from the Mott-Gurney equation $J=9 \cdot \varepsilon \cdot \varepsilon_{0} \cdot \mu \cdot V^{2} / 8 \cdot L^{3}$ ( $\varepsilon$ and $\varepsilon_{0}$ dielectric permittivity and dielectric constant, respectively).

The absorbance spectra of the spin-coated PDI (Fig. 4c) display a strong absorbance in the visible region with two main peaks appearing at $670 \mathrm{~nm}$ and $440 \mathrm{~nm}$. For the same molar concentrations of $10 \mathrm{mM}$, the 1,6 pattern (PDI-2) shows higher absorbance intensity and blue shifting as compared to the 1,7 (PDI-1). Moreover, PDI-2 displays the appearance of a shoulder at $550 \mathrm{~nm}$.

The absorbance spectra of the PDIs deposited on perovskite (Fig. 5a) suggest that the PDI deposition has negligible influence on the absorption in the broad part of the spectrum. Despite intense absorption of PDI as such, its characteristic signature peaks are suppressed in the presence of perovskite absorption. No new absorbance band appeared with the usage of HTM layers.

For steady-state photoluminescence (PL) spectra, the samples were deposited on quartz substrates (Fig. 5b). The $\mathrm{MAPbI}_{3}$ layer exhibits a strong PL emission peak centered at $778 \mathrm{~nm}$, while bilayer (perovskite/HTM) showed a significant quenching in PL as compared to perovskite, suggesting the separation and extraction of charge carriers. It can be deduced from Fig. $5 b$ that the maximum PL quenching occurs with the doped PDI-1, which is on par with doped Spiro-OMeTAD, pointing out that the free carriers generated by light irradiation are separated and transferred efficiently. The maximum quenching in PL 
Fig. 1 DFT-B3LYP fron-

tier molecular orbitals with calculated HOMO and LUMO energy levels computed for PDI-1 (a, b) and PDI-2 (c, d) (isosurface value $=0.02$ )
Fig. 2 DFT-B3LYP optimized geometries for PDI-1 and PDI2 (6-311G* basis set) and core twist angle values a)

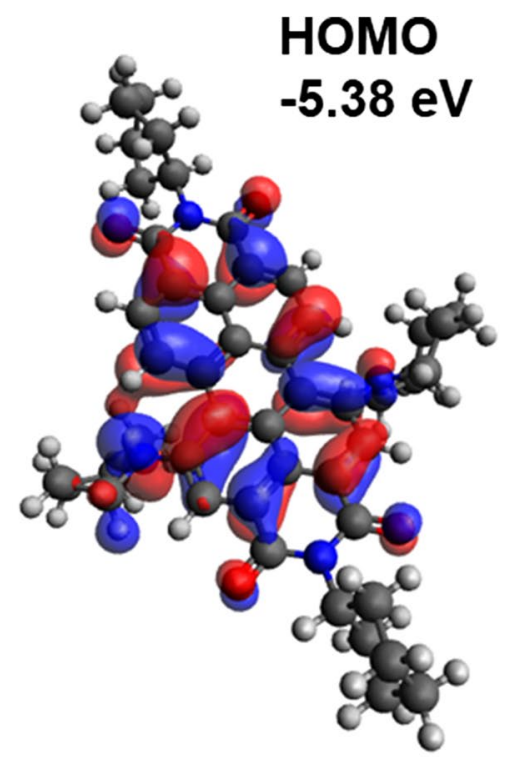

c)
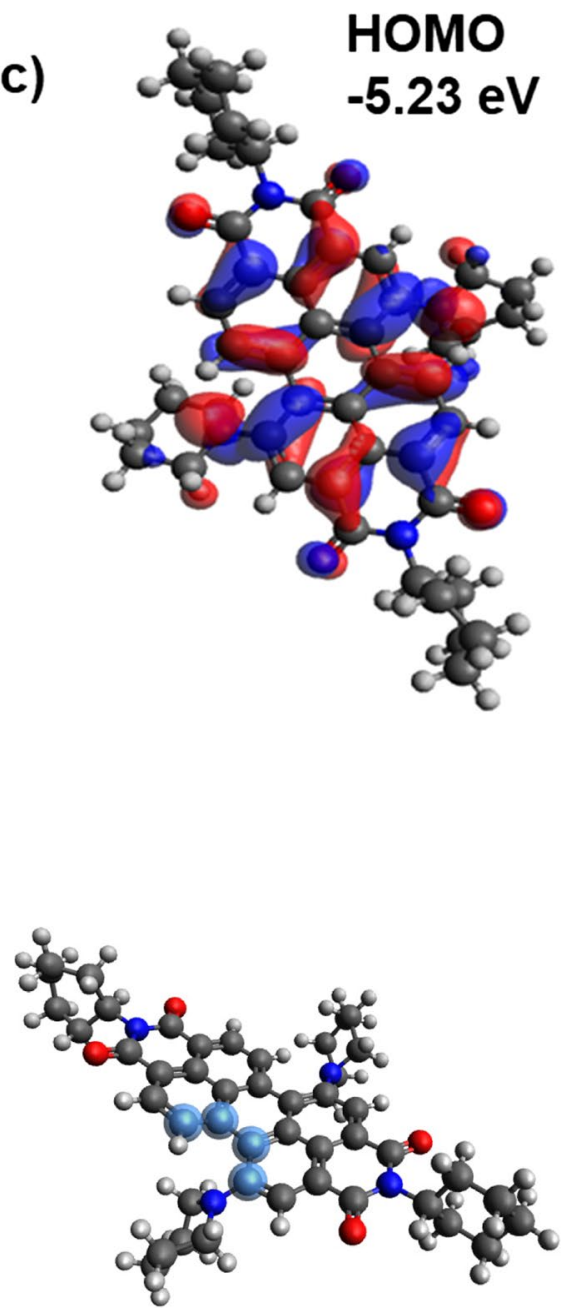

PDI-1 core twist angle (dihedral $\mathrm{C}_{6}-\mathrm{C}_{7}$ ) b)

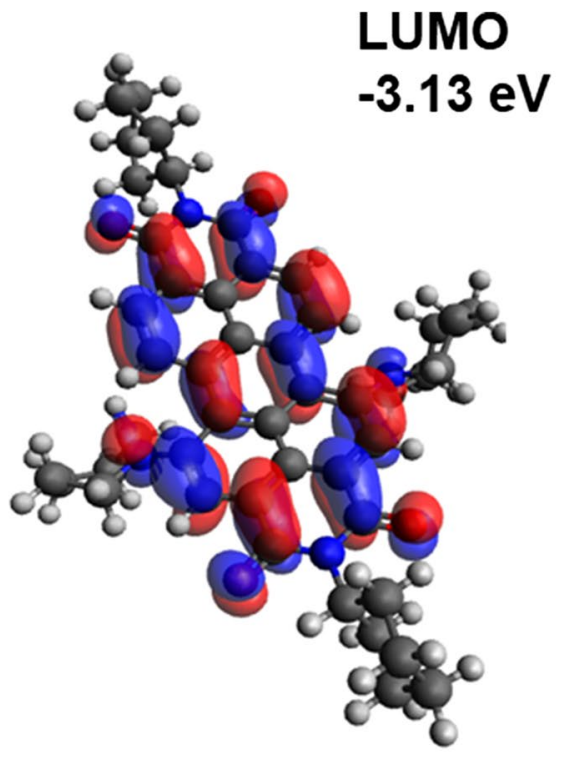

d)

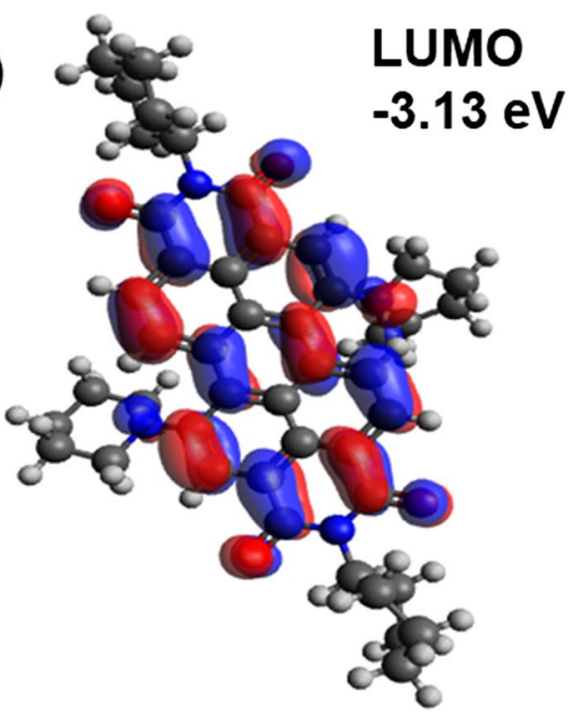

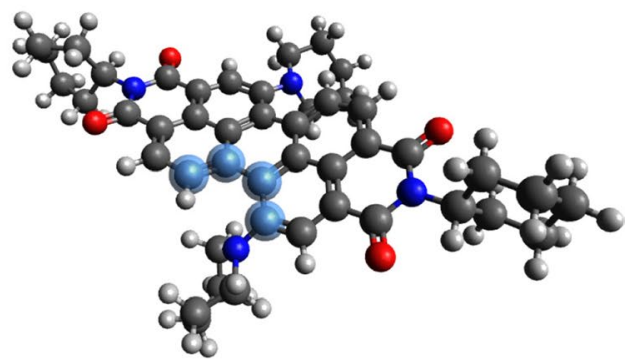

PDI-2 core twist angle (dihedral $\left.\mathrm{C}_{6}-\mathrm{C}_{7}\right)=$ $20.4^{\circ}$ 
a)

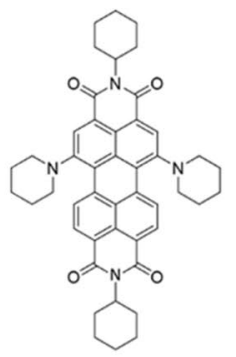

PDI 1

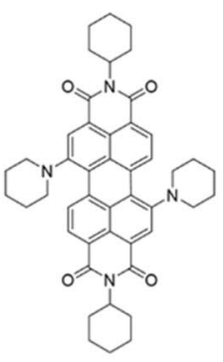

PDI 2

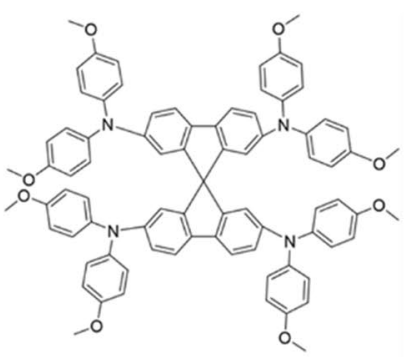

Spiro-OMeTAD

b)

FTO $\mathrm{TiO}_{2} \quad \mathrm{MAPbl}_{3}$ Spiro PDI-1 PDI-2 Au
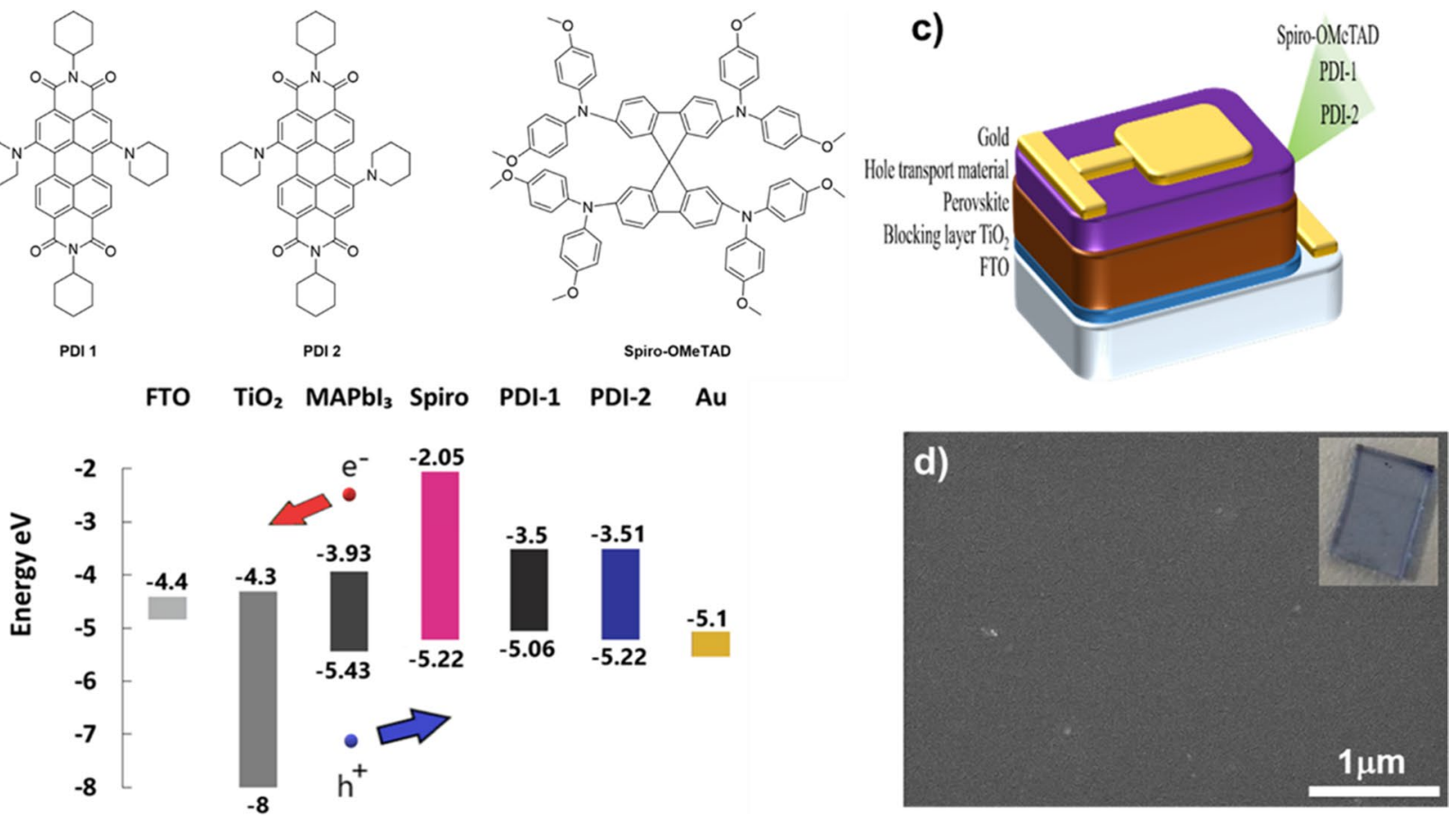

Fig. 3 a Structure of the PDIs and Spiro-OMeTAD, b energy levels of the HTMs and other constituents in the device, $\mathbf{c}$ architecture of fabricated PSCs, and d scanning electron microscopy image of PDI-2

deposited on standard glass; inset represents the visual image of PDI$\mathbf{2}$ deposited on FTO glass
Fig. 4 a $J-V$ curve from the curve of PDI-2 based HTMs, and $\mathbf{c}$ absorbance spectra of the PDI films conductivity of PDI-2, b $J-V$
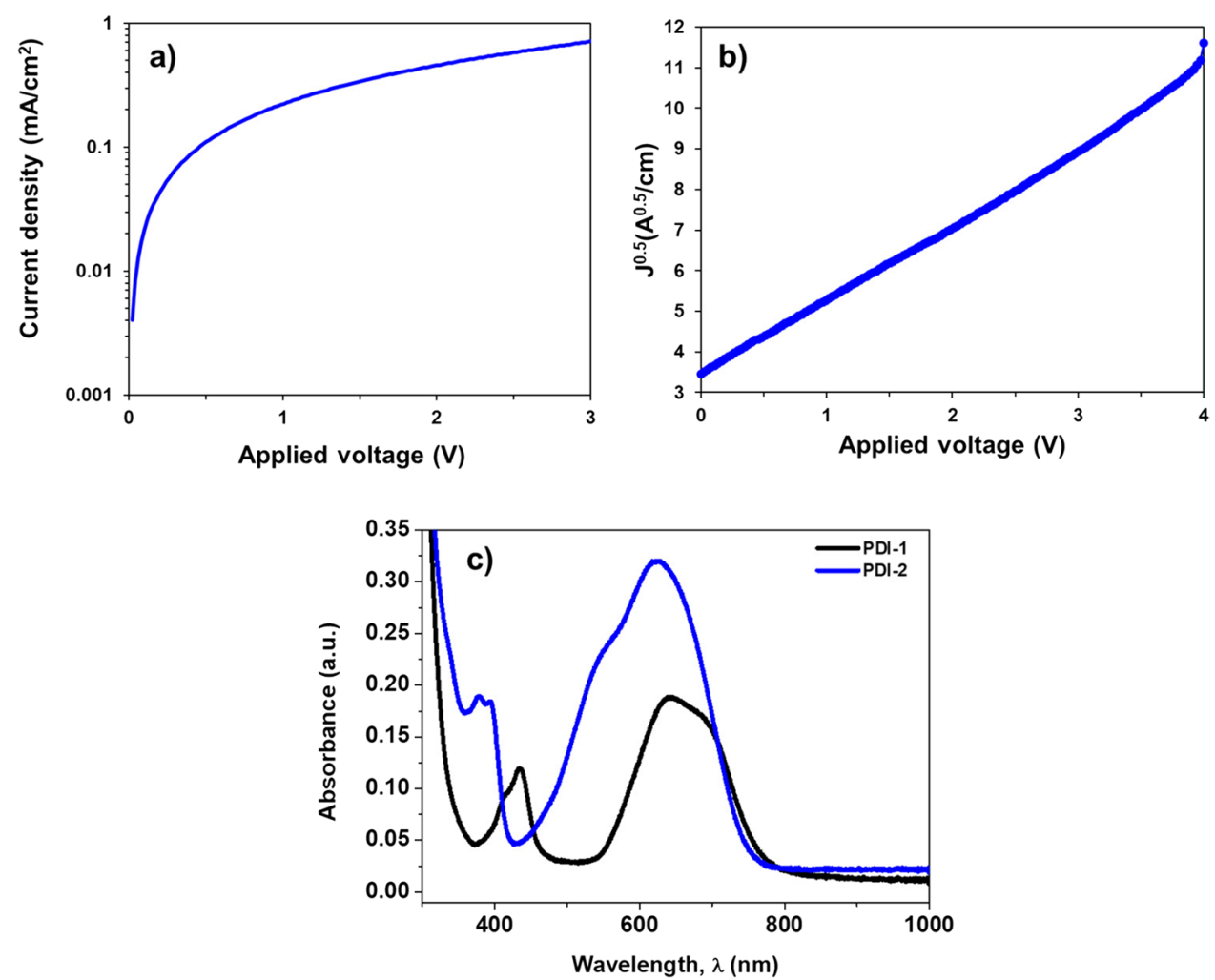

intensity by doped PDI-1 signals maximized hole extracSurface defects, including vacancies and interstitials, are tion rate induced by high charge carrier concentration. present at the surface and grain boundaries. Such defects 
Fig. 5 a Absorption spectra of PDIs atop of perovskites and b steady-state PL emission measured at $600 \mathrm{~nm}$ excitation of pristine perovskite and perovskite with the HTMs
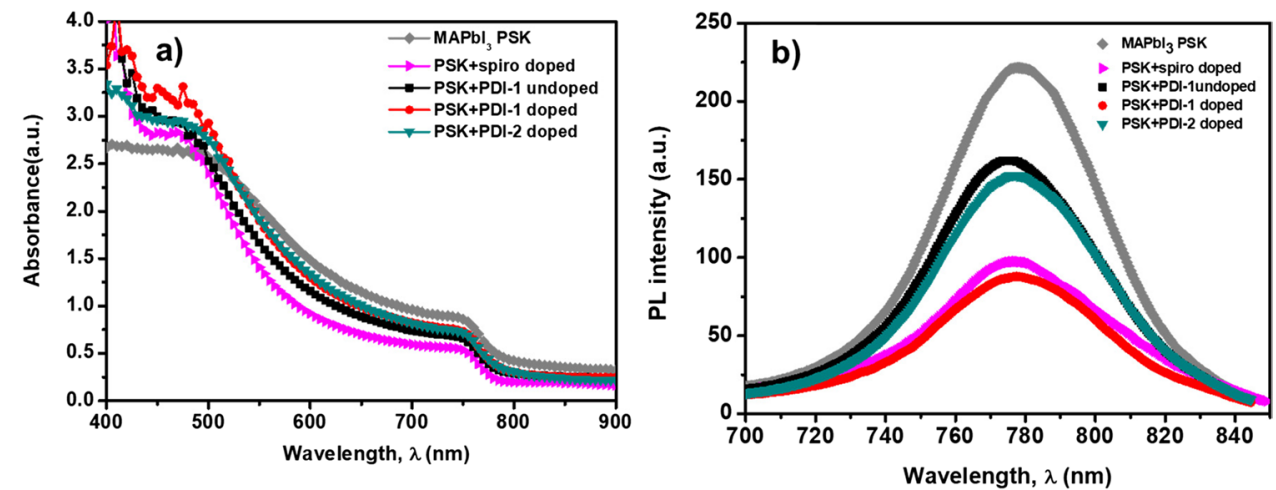

and charges present in under-coordinated $\mathrm{Pb}^{2+}$ at the interface, interacted with organic materials, and blue shifted the PL emission.

However, in the fabricated PSCs, other factors, such as carrier recombination at perovskite/HTM and HTM/Au interfaces, play a dominant role in the device performance.

The surface properties of the PDI (Fig. 6) were studied by performing goniometry experiments to determine the surface contact angle, and expectedly dopant-free HTMs gave a higher contact angle value. We noted that samples with PDIs showed higher hydrophobic character than those with SpiroOMeTAD. The contact angle value for the pristine perovskite lies at $67^{\circ}$, and with a pristine Spiro-OMeTAD layer, this value increases significantly to $88^{\circ}$. However, pristine Spiro-OMeTAD is not effective for device fabrication and requires doping. Doping of Spiro-OMeTAD decreases the contact angle value, due to the use of hygroscopic lithium salt. In the case of PDI, the pristine and doped contact angle value remains largely unaltered and displays hydrophobicity, while undoped PDI-2 gave a marginally higher value. Both doped and undoped PDIs showed improved surface properties, signaling increase moisture barrier properties, which are effective to increase the stability of PSCs.

Pristine and doped PDI-1 and PDI-2 were spin-coated (10 mM concentration) as HTM for PSCs fabrication. In the case where the dopant is used for PDIs, the classical dopant/ additives $\{$ LiTFSI $(50 \%) / t$-BP $(330 \%)\}$ were used, and their concentrations were kept similar as for Spiro-OMeTAD. $\mathrm{MAPbI}_{3}$ was used as an active layer due to the aligned energetic level to the PDIs. Arguably, the use of dopants (LiTFSI and $t$-BP) can enhance the $\mathrm{p}$-type conductivity and decrease the resistivity of the HTM. However, the hygroscopic character of lithium salts accelerates the degradation in PSCs due to the poisoning of the interface between Spiro-OMeTAD and the perovskite as well as migration of lithium ions into the bulk of perovskites [30]. Doping has a low impact on the PDI surface properties, which may be due to its lower thickness in comparison with Spiro-OMeTAD.

It can be deduced (Table 1) that PDI as compared to doped Spiro-OMeTAD yielded a drop in the current density $\left(J_{\text {sc }}\right)$, while comparable $V_{o c}$ and a slight decrease in the FF due to higher series resistance. Competitive $V_{o c}$ is indicative
Fig. 6 Contact angle values of perovskites without and with different HTMs (doped or undoped)

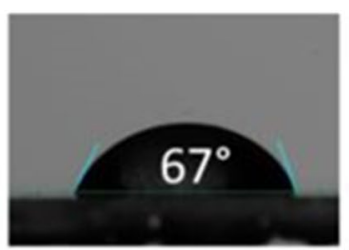

Perovskite

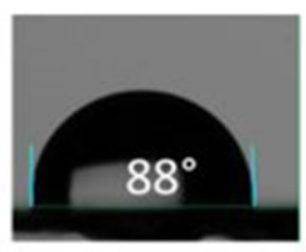

Spiro-OMeTAD (undoped)

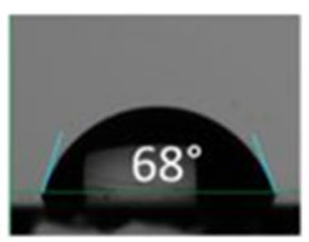

Spiro-OMeTAD (doped)

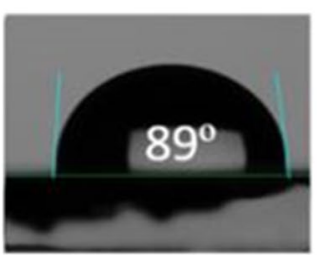

PDI-1 (undoped)

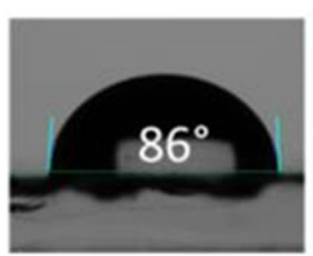

PDI-1 (doped)

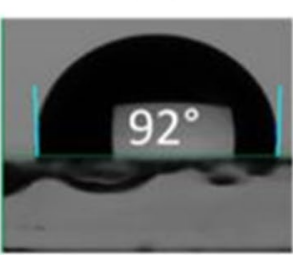

PDI-2 (undoped)

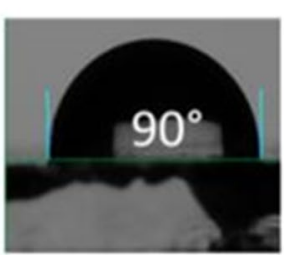

PDI-2 (doped) 
Table 1 Characteristic parameters of $J$ - $V$ measurement

\begin{tabular}{lllllll}
\hline Sample & $V_{\mathrm{oc}}(\mathrm{mV})$ & $J_{\mathrm{sc}}\left(\mathrm{mA} / \mathrm{cm}^{2}\right)$ & FF $(\%)$ & PCE $(\%)$ & \multicolumn{2}{c}{$\begin{array}{l}\text { Series resist- } \\
\text { ance }(\Omega)\end{array}$} \\
\hline PDI-1 & 929 & 13.44 & 66.8 & $8.34(6.96)$ & 88.68 & 3137 \\
PDI-2 & 880 & 12.21 & 64.7 & $6.98(4.73)$ & 101.30 & 5960 \\
Spiro-OMeTAD & 700 & 15.27 & 36.3 & 3.92 & 125 & 626 \\
PDI-1 (doped) & 923 & 10.35 & 79.5 & $7.69(7.05)$ & 77.17 & 4305 \\
PDI-2 (doped) & 870 & 7.32 & 71.2 & $4.55(3.75)$ & 109.23 & 13576 \\
Spiro-OMeTAD (doped) & 1038 & 20.98 & 76.9 & $16.75(15.27)$ & 22.95 & 107805 \\
\hline
\end{tabular}

*Average values of PCE are expressed into brackets

of aligned energy level with the perovskite, allowing efficient separation of the electron-hole pairs at the interface. The shunt resistance in PDIs based PSCs is prevailing, showing fewer shunt paths of recombination; however, it evolves with voltage (Fig. 7a). Suggesting recombination paths at the perovskite/PDI-1 interface is dominant at lower voltages, due to the rotations or deformations of the PDIs at the interface. Reduction in FF is mainly due to an increased series resistance, which we ascribed to the electrical conductivity of PDIs.

The generated photocurrent was investigated by EQE spectra (Fig. 7b), which reveal direct relation with the drop in the $J_{S C}$. At the lower wavelength $(300-500 \mathrm{~nm})$ range, which represents the $\mathrm{TiO}_{2}$ /perovskite interface, charge collections trends are similar. However, at a higher wavelength $(550-850 \mathrm{~nm})$, there is a steep drop in the charge generation. We attribute a partial loss of current in this region to a charge accumulation at the perovskite/HTM interface, as the longer wavelengths will be absorbed closer to the HTM. These properties are similar for both doped and undoped PDI films, suggesting doping does not influence the electrical properties to a larger extent. In the case of Spiro-OMeTAD, the large size of the molecule can facilitate oxidation, while the two-dimensional nature of PDIs inhibits doping, and owing to its two-dimensional molecular design, PDIs will not similarly coordinate with the dopant as of Spiro-OMeTAD.
Pristine PDIs gave an improved PCE as compared to Spiro-OMeTAD, and PDI-1 yielded a PCE of $8.34 \%$. Pristine Spiro-OMeTAD possesses inferior electrical properties which affect the LUMO level, which is below the valence band of perovskite, reducing the built-in potential and thus the photo-voltage. Additionally, the required thickness for Spiro-OMeTAD is much higher than for PDI, which affects the fill factor [31]. The small molecular size of PDIs will not coordinate in the same way with the dopants. The development of dopant-free HTM is paramount for PSCs fabrication, as the use of dopants triggers unwanted side reactions and decomposition.

Pristine PDIs were deposited from $10 \mathrm{mM}$ concentrations as compared to typically $70 \mathrm{mM}$ for Spiro-OMeTAD; this will cut down materials consumption and cost. To decipher the carrier recombination and origin of parasitic light absorption, we performed electrochemical impedance spectroscopy (EIS) measurements. Figure 8a and b represent Nyquist and Bode plots for the fabricated PSCs under light illumination. In this regime, the carrier concentration is equilibrated by the photo-generated charge, the movement is dominated by diffusion, and a non-ideal process such as charge accumulation can be identified. We noted dissimilar profiles among Spiro-OMeTAD, PDI-1, and PDI-2. In the case of Spiro-OMeTAD and PDI-1 (pristine or doped), only a semicircle appears, while in the case of PDI-2 (pristine or doped), two semicircles appear at different frequencies,
Fig. 7 a $J-V$ curves of pristine PDI-1 and PDI-2 and doped Spiro-OMeTAD and $\mathbf{b}$ EQE of the PDIs and doped SpiroOMeTAD
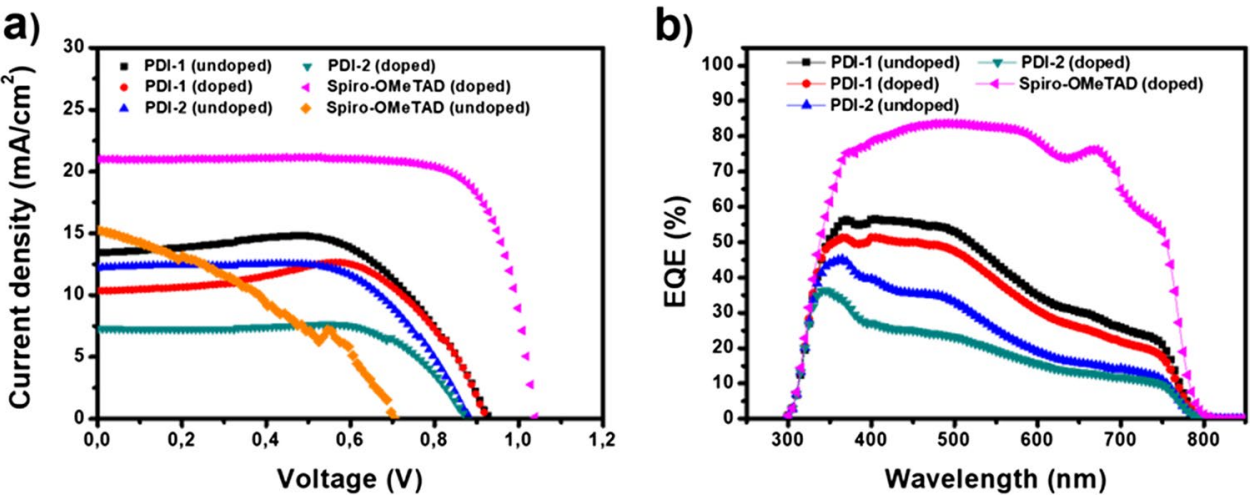

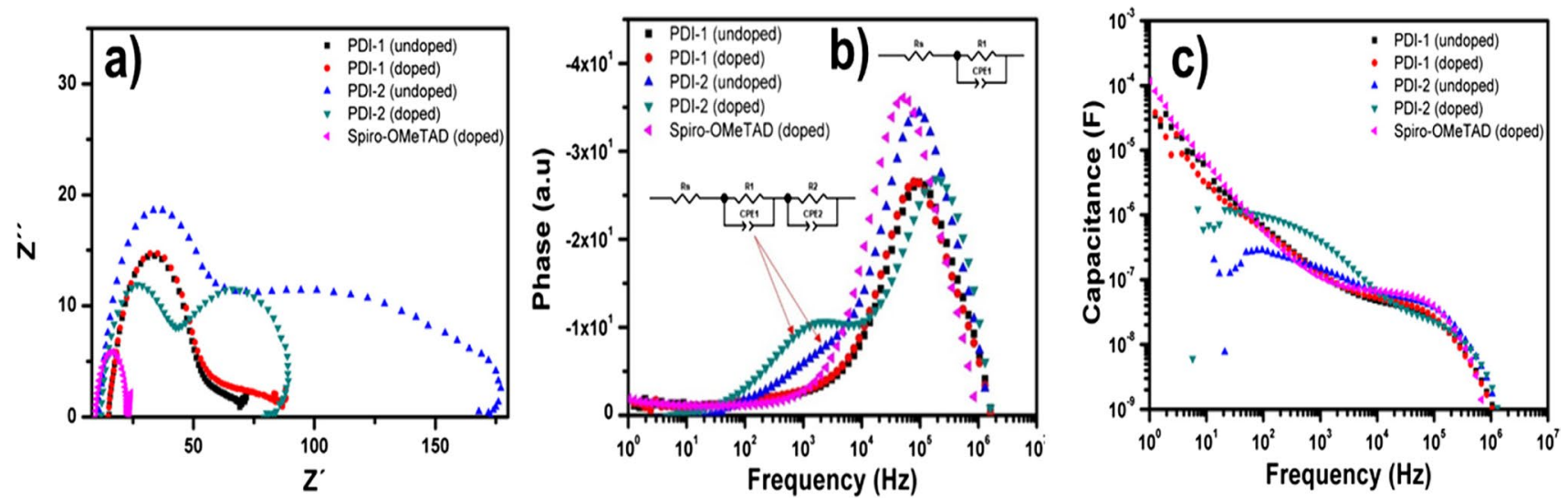

Fig. 8 a Nyquist plot, b Bode plot, and c capacitance vs frequency plots of PSCs using different HTMs (doped and undoped)

suggesting the change in device kinetics processes. Bulk recombination in the perovskite is a fast process that occurs in the range of $10^{5}-10^{6} \mathrm{~Hz}$; however, in the case of PDI-2, an additional process appears at $10^{3} \mathrm{~Hz}$. The slower processes (in the range of $10^{-1} \mathrm{~Hz}$ ) in PSCs are attributed to a charge accumulation together with the ionic motion that induces hysteresis behavior. Figure $8 \mathrm{c}$ depicts the capacitance as a function of frequency; the trend illustrates the typical behavior of PSCs, i.e., characterized by a plateau at the mid-frequency region that can be associated with the bulk capacitance of the perovskite. On the contrary, PDI2 based PSCs showed a different behavior, with an increment in the capacitance at mid-frequency. We ascribed this behavior to the accumulation of charge at the perovskite/ HTM interface, increasing the charge recombination at the interface and thus limiting its performance as compared to PDI-1. This is following the PL properties.

\section{Conclusions}

We report the use of di(piperidinyl)perylenediimide as a hole-selective layer in $\mathrm{MAPbI}_{3}$-based solar cells. The position of substituents influences the electro-optical properties, energetic levels, and kinetics of the devices. The substituents at the bay positions of 1,6- and 1,7-dipiperidinyl PDI impact photovoltaic properties. Our study suggests that PDIs are potentially attractive candidates as hole-selective layers; furthermore, by tuning the energetic level with rational substituents, the performance in perovskite solar cells can be optimized. The fabricated solar cells in an $n-i-p$ configuration, using an extremely thin film of pristine PDIs as hole transport materials, yielded competitive $V_{o c}$ and $F F$, while limited photocurrent as compared to doped Spiro-OMeTAD was noted. Suggesting molecular engineering in PDI for band alignment and carrier concentration increase is paramount for performance enhancement.

\section{Experimental part}

\subsection{Chemicals}

The chemicals (dimethylsulfoxide, anhydrous chlorobenzene, and lithium bis(trifluoromethylsulfonyl)imide (LiTFSI)) are commercial products and procured either from Sigma-Aldrich or Acros organics. For comparative purpose, the classical HTM, i.e., 2,2' 7,7'-tetrakis( $N, N$-di-pmethoxyphenyamine)-9,9-spirobifluorene (Spiro-OMeTAD) was acquired from Merck KGaA. Methylammonium iodide (MAI) was obtained from Dyesol while $\mathrm{PbI}_{2}$ from Tokyo Chemical Industry (TCI). PDI-1 and PDI-2 were synthesized according to our previous reports [26].

\subsection{Device fabrication}

For solar cells fabrication, FTO (NSG10) was used as a substrate. Before its usage, it was cleaned in a three-step sequential sonication treatment with $2 \%$ Hellmanex solution, acetone, and isopropanol, followed by UV ozone treatment for $20 \mathrm{~min}$. The substrates were then heated to $500{ }^{\circ} \mathrm{C}$ to remove any organic components on the surface. Next, a compact blocking layer of $\mathrm{TiO}_{2}$ was deposited onto the FTO substrate by spray pyrolysis, using a dilution of $1 / 19 \mathrm{~mL}$ of titanium (IV) diisopropoxide bis(acetylacetonate) solution in ethanol keeping them at $500{ }^{\circ} \mathrm{C}$ for $30 \mathrm{~min}$. Once the substrates were cooled down to room temperature, a diluted $\mathrm{TiO}_{2}$ dispersion in ethanol ratio 1:8 (w/v) $(30 \mathrm{NRD}$ from Dyesol) was deposited by spin-coating at $2000 \mathrm{rpm}$ (1000 rpm/s acceleration) for $20 \mathrm{~s}$, followed by a progressive heating step until $500{ }^{\circ} \mathrm{C}$ for $30 \mathrm{~min}$. Stoichiometric precursor solutions $(1.25 \mathrm{M})$ were prepared by mixing MAI and $\mathrm{PbI}_{2}$ in dimethylsulfoxide (DMSO) and kept under stirring at $70{ }^{\circ} \mathrm{C}$ overnight to dissolve $\mathrm{PbI}_{2}$ completely. The perovskite was deposited in a two-step spin-coating process (first step $1000 \mathrm{rpm}$ for $10 \mathrm{~s}$; second step $3500 \mathrm{rpm}$ for $30 \mathrm{~s}$ ). During

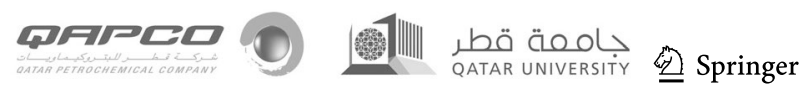


the second step, $110 \mu \mathrm{L}$ of chlorobenzene was dripped onto the substrates $10 \mathrm{~s}$ before the end of the program. The substrates were then annealed at $100{ }^{\circ} \mathrm{C}$ for $40 \mathrm{~min}$. On acquiring room temperature, HTM was deposited, and for this, doped and undoped PDI-1 and PDI-2 (10 mM) and doped Spiro-OMeTAD $(70 \mathrm{mM})$ dissolved in chlorobenzene were spun coated at $4000 \mathrm{rpm}$ for $30 \mathrm{~s}$. The resultant thickness of PDIs was $\sim 20 \mathrm{~nm}$. The molar percentage of dopants to the HTM used in this work was $50 \%$ of LiTFSI and $330 \%$ of 4-tert-butylpyridine (t-BP). Perovskite and hole transport layers were prepared inside an argon-filled glove box $\left(\mathrm{H}_{2} \mathrm{O}\right.$ level: $<1 \mathrm{ppm}$ and $\mathrm{O}_{2}$ level $<10 \mathrm{ppm}$ ). Finally, $70 \mathrm{~nm}$ of gold as electrical contact was thermally evaporated to close the circuit.

\subsection{Device characterization}

The undoped PDI-1 and PDI-2 films were deposited on quartz, and the absorption spectra were registered with the help of a UV-Vis spectrophotometer (Varian Cary 60). For absorption and emission studies, firstly the perovskite layer was spin-coated on the quartz substrate followed by HTM deposition. Steady-state photoluminescence (PL) studies were made with the help of a fluorescence spectrophotometer (LS55 Perkin Elmer). For contact angle (Ossila, UK) measurements, a $3 \mu \mathrm{L}$ drop of water was dripped on top of perovskite/HTM samples. The curve was fitted to the edge of the droplet, using the contact angle software, and the respective angle was calculated. Scanning electron microscopy images were recorded with the help of JEOL JSM-6400. The highest occupied molecular orbital (HOMO) and lowest unoccupied molecular orbital (LUMO) were elucidated with the help of cyclic voltammetry. The HOMO and LUMO levels were estimated from the onset values for the reduction and oxidation potentials through the equations: $\mathrm{LUMO}=-\left|\mathrm{E}_{\text {red1 }}\left(\mathrm{vs} \mathrm{Fc} / \mathrm{Fc}^{+}\right)+4.80\right| \mathrm{eV}$ and $\mathrm{HOMO}=\mathrm{LUMO}-E_{\mathrm{g}} \mathrm{eV}$. Ferrocene/ferrocenium ion $\left(\mathrm{Fc} / \mathrm{Fc}^{+}\right)$was used as the external redox couple, and $4.8 \mathrm{eV}$ below the vacuum was established as a reference.

Current density-voltage $(J-V)$ curves were logged with the help of a Keithley 2400 source meter. For $J$ - $V$ curves under illumination, AM $1.5 \mathrm{G}, 100 \mathrm{~mW} / \mathrm{cm}^{2}$ illumination from a Class AAA, 450 W solar simulator (ORIEL, 94,023 A) was used. Light output power was calibrated using NREL certified calibrated mono-crystalline silicon solar cell. A black metal mask $\left(0.16 \mathrm{~cm}^{2}\right)$ was placed over the squareshaped device (active area $0.5 \mathrm{~cm}^{2}$ ) to reduce the influence of scattered light. An optimized scan rate of $10 \mathrm{mV} / \mathrm{s}$ was used for measuring the devices to calculate the accurate efficiencies without having hysteresis effects. For IPCE measurements, a $75 \mathrm{~W}$ Xenon and $100 \mathrm{~W}$ Quartz halogen (QTH) lamp coupled to a $300 \mathrm{~mm}$ focal length, triple grating monochromator (TMC300), while a Bentham 610 power meter was used.

The frequency response of the devices was acquired by performing electrochemical impedance spectroscopy (EIS); for this, $20 \mathrm{mV}$ perturbation in the $10^{6}-10^{-1} \mathrm{~Hz}$ range was made using a potentiostat (SP300, Biologic) in a faraday chamber to avoid any extra signal. The Fermi level of the semiconductor was modified by illuminating the samples with a white LED over a wide range of DC light intensities, starting from the open-circuit voltage under AM 1.5 G, $100 \mathrm{~mW} / \mathrm{cm}^{2}$ illumination and decrease the intensity every $50 \mathrm{mV}$. To avoid voltage drop due to series resistance, EIS measurements were performed at the open-circuit potential, the Fermi level (related to the open-circuit voltage) being fixed by the DC illumination intensity. The EC-Lab software was used to generate EIS data, whereas Z-view equivalent circuit modeling software (Scribner) was used to fit the spectra.

Supplementary Information The online version contains supplementary material available at https://doi.org/10.1007/s42247-021-00317-z.

Funding Open Access funding provided thanks to the CRUE-CSIC agreement with Springer Nature. This work has received funding from the European Union H2020 Programme under European Research council Consolidator grant [MOLEMAT, 726360]. The group at Universidad Miguel Hernández thanks the financial support provided by the European Regional Development Fund "A way to make Europe" and the Spanish Ministerio de Ciencia e Innovación/Agencia Estatal de Investigación through projects CTQ2016-77039-R and PID2019109200 GB-I00 (MCI/AEI/FEDER, UE). MS thank the National Research grant "Juan de la Cierva" [FJCI-2017-31761].

Availability of data and material There is no supplementary material.

Code availability Not applicable.

\section{Declarations}

Conflict of interest The authors declare no competing interests.

Open Access This article is licensed under a Creative Commons Attribution 4.0 International License, which permits use, sharing, adaptation, distribution and reproduction in any medium or format, as long as you give appropriate credit to the original author(s) and the source, provide a link to the Creative Commons licence, and indicate if changes were made. The images or other third party material in this article are included in the article's Creative Commons licence, unless indicated otherwise in a credit line to the material. If material is not included in the article's Creative Commons licence and your intended use is not permitted by statutory regulation or exceeds the permitted use, you will need to obtain permission directly from the copyright holder. To view a copy of this licence, visit http://creativecommons.org/licenses/by/4.0/. 


\section{References}

1. Y. Fan, K. Ziabrev, S. Zhang, B. Lin, S. Barlow, S.R. Marder, ACS Omega 2, 377-385 (2017)

2. F. Würthner, Chem. Commun. 4, 1564-1579 (2004)

3. C. Huang, S. Barlow, S.R. Marder, J. Org. Chem. 76, 2386-2407 (2011)

4. A. Nowak-Król, F. Würthner, Org. Chem. Front. 6, 1272-1318 (2019)

5. Z. Liu, Y. Wu, Q. Zhang, X. Gao, J. Mater. Chem. A 4, 1760417622 (2016)

6. F. Fernández-Lázaro, N. Zink-Lorre, Á. Sastre-Santos, J. Mater. Chem. A 4, 9336-9346 (2016)

7. R. Muñoz-Mármol, N. Zink-Lorre, J.M. Villalvilla, P.G. Boj, J.A. Quintana, C. Vázquez, A. Anderson, M.J. Gordon, A. Sastre-Santos, F. Fernández-Lázaro, M.A. Díaz-García, J. Phys. Chem. C 122, 24896-24906 (2018)

8. M. Ball, B. Zhang, Y. Zhong, B. Fowler, S. Xiao, F. Ng, M. Steigerwald, C. Nuckolls, Acc. Chem. Res. 52, 1068-1078 (2019)

9. H. Liu, H. Wang, G. Liu, S. Pu, H. Zhang, Tetrahedron 75, 19881996 (2019)

10. N. Zink-Lorre, E. Font-Sanchis, Á. Sastre-Santos, F. FernándezLázaro, Chem. Commun. 56, 3824-3838 (2020)

11. J.L. Wu, W.K. Huang, Y.C. Chang, B.C. Tsai, Y.C. Hsiao, C.Y. Chang, C.T. Chen, C.T. Chen, J. Mater. Chem. A 5, 12811-12821 (2017)

12. K. Jiang, F. Wu, H. Yu, Y. Yao, G. Zhang, L. Zhu, H. Yan, J. Mater. Chem. A 6, 16868-16873 (2018)

13. Z. Luo, F. Wu, T. Zhang, X. Zeng, Y. Xiao, T. Liu, C. Zhong, X. Lu, L. Zhu, S. Yang, C. Yang, Angew. Chemie Int. Ed. 58, 8520-8525 (2019)

14. D. Zou, F. Yang, Q. Zhuang, M. Zhu, Y. Chen, G. You, Z. Lin, H. Zhen, Q. Ling, Chemsuschem 12, 1155-1161 (2019)
15. J. Huang, Z. Gu, X. Zhang, G. Wu, H. Chen, J. Alloys Compd. 767, 870-876 (2018)

16. J. Yang, C. Liu, C. Cai, X. Hu, Z. Huang, X. Duan, X. Meng, Z. Yuan, L. Tan, Y. Chen, Adv. Energy Mater. 9, 1900198 (2019)

17. Q. Zhou, Y. Gao, C. Cai, Z. Zhang, J. Xu, Z. Yuan, P. Gao, Angew. Chem. Int. Ed. 60, 8303-8312 (2021)

18. J. Das, R. BhaskarKanthSiram, D. Cahen, B. Rybtchinski, G. Hodes, J. Mater. Chem. A 3, 20305-20312 (2015)

19. C. Zhao, Y. Zhang, R. Li, X. Li, J. Jiang, J. Org. Chem. 72, 2402 $2410(2007)$

20. G. Goretzki, E.S. Davies, S.P. Argent, W.Z. Alsindi, A.J. Blake, J.E. Warren, J. McMaster, N.R. Champness, J. Org. Chem. 73, 8808-8814 (2008)

21. M.J. Lin, Á.J. Jiménez, C. Burschka, F. Würthner, Chem. Commun. 48, 12050-12052 (2012)

22. N. Zink-Lorre, E. Font-Sanchis, Á. Sastre-Santos, F. FernándezLázaro, Org. Biomol. Chem. 14, 9375-9383 (2016)

23. D. Gutiérrez-Moreno, A. Sastre-Santos, F. Fernández-Lázaro, Org. Chem. Front. 5, 1830-1834 (2018)

24. N. Zink-Lorre, E. Font-Sanchis, Á. Sastre-Santos, F. FernándezLázaro, Dye. Pigment. 127, 9-17 (2016)

25. L. Fan, Y. Xu, H. Tian, Tetrahedron Lett. 46, 4443-4447 (2005)

26. D. Gutiérrez-Moreno, Á. Sastre-Santos, F. Fernández-Lázaro, Org. Chem. Front. 6, 2488-2499 (2019)

27. G. Rauch, S. Höger, Chem. Commun. 50, 5659-5661 (2014)

28. J. Feng, D. Wang, H. Wang, D. Zhang, L. Zhang, X. Li, J. Phys. Org. Chem. 24, 621-629 (2011)

29. H.Y. Tsai, K.Y. Chen, Dye. Pigment. 96, 319-327 (2013)

30. J.A. Dawson, A.J. Naylor, C. Eames, M. Roberts, W. Zhang, H.J. Snaith, P.G. Bruce, M. Saiful Islam, ACS Energy Lett. 2, 18181824 (2017)

31. W. Luo, C. Wu, D. Wang, Z. Zhang, X. Qi, X. Guo, B. Qu, L. Xiao, Z. Chen, Org. Electron. 74, 7-12 (2019) 